\title{
Prognostic value of DCE-CT-derived blood volume and flow compared to core biopsy microvessel density in patients with metastatic renal cell carcinoma
}

Aska Drljevic-Nielsen ${ }^{1,2^{*}}$, Finn Rasmussen ${ }^{2}$, Patricia Switten Nielsen ${ }^{3}$, Christina Stilling ${ }^{3}$, Kennet Thorup ${ }^{2}$, Jill Rachel Mains ${ }^{2}$, Hans Henrik Torp Madsen ${ }^{2}$ and Frede Donskov ${ }^{1}$

\begin{abstract}
Background: Angiogenesis is prominent in metastatic renal cell carcinoma (mRCC). We compared two angiogenesis assessment methods: dynamic contrast-enhanced computed tomography (DCE-CT)-derived blood volume (BV) and blood flow (BF) and core biopsy microvessel density (MVD).
\end{abstract}

Methods: As planned in DaRenCa Study-1 study, DCE-CT and core biopsy were performed from the same tumour/ metastasis at baseline. MVD was assessed by CD34 immunostaining in tumour (CD34-index T $_{\text {) or tumour including }}$ necrosis $\left(C D 34-i n d e x_{T N}\right)$. BV and BF were assessed using the DCE-CT software. Overall survival (OS) and progressionfree survival (PFS) were assessed by Kaplan-Meier analysis. Spearman coefficient (rho) tested the correlation between MVD and BV, BF, or CT density (HU).

Results: At baseline, 25 patients had analysable scans and tissue. $\mathrm{BV}_{\text {deconv, }} \mathrm{BV}_{\text {Patlak, }}$ and $\mathrm{BF}_{\text {deconv }}>$ median were associated with favourable OS (43.2 versus 14.6 months, $p=0.002 ; 31.6$ versus 20.2 months, $p=0.015$; and 31.6 versus 24.5 months, $p=0.019)$. CD34-index and CD34-index $x_{\text {TN }}$ did not correlate with age $(p=0.543)$, sex ( $p=$ $0.225)$, treatment $(p=0.848)$, International mRCC Database Consortium category $(p=0.152)$, synchronous versus metachronous metastatic disease $(p=0.378)$, or tumour volume $(p=0.848)$. CD34-index or CD34-index TN $_{\text {T }}>$ median was not associated with PFS ( $p=0.441$ and $p=0.854$, respectively) or OS ( $p=0.987$ and $p=0.528$, respectively). CD34-index or CD34-index $x_{T N}$ was not correlated with BV, BF, or HU (rho 0.20-0.26).

Conclusions: Differently from MVD, DCE-CT-derived BV and BF had prognostic impact and may better reflect angiogenesis in $\mathrm{mRCC}$.

Trial registration: NCT01274273

Keywords: Blood volume, Carcinoma (renal cell), Microvascular density, Prognosis, Tomography (x-ray computed)

\footnotetext{
*Correspondence: askadrlj@rm.dk

'Department of Oncology, Aarhus University Hospital (AUH), Palle

Juul-Jensens Blvd. 99, 8200 Aarhus N, Denmark

${ }^{2}$ Department of Radiology, Aarhus University Hospital (AUH), Palle

Juul-Jensens Blvd. 99, 8200 Aarhus N, Denmark

Full list of author information is available at the end of the article
}

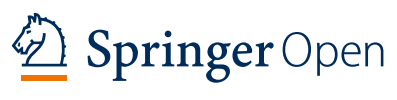

(c) The Author(s). 2021 Open Access This article is licensed under a Creative Commons Attribution 4.0 International License, which permits use, sharing, adaptation, distribution and reproduction in any medium or format, as long as you give appropriate credit to the original author(s) and the source, provide a link to the Creative Commons licence, and indicate if changes were made. The images or other third party material in this article are included in the article's Creative Commons licence, unless indicated otherwise in a credit line to the material. If material is not included in the article's Creative Commons licence and your intended use is not permitted by statutory regulation or exceeds the permitted use, you will need to obtain permission directly from the copyright holder. To view a copy of this licence, visit http://creativecommons.org/licenses/by/4.0/. 


\section{Key points}

- Angiogenesis is prominent in patients with metastatic renal cell carcinoma (mRCC).

- Angiogenesis assessed by microvascular density (MVD) was compared to functional imaging.

- Dynamic contrast-enhanced computed tomographyderived functional parameters blood volume (BV) and blood flow (BF) showed strong prognostic ability.

- MVD did not show prognostic potential or association to patient characteristics.

- MVD did not correlate to BV or BF in mRCC.

\section{Background}

Clear cell renal cell carcinoma (ccRCC) is the most frequent kidney cancer histological subtype. Genetic mutations in the Von Hippel Lindau tumour suppressor gene on chromosome three in the proximal convoluted tubule epithelium [1] lead to increased angiogenesis, and thus a highly vascularised tumour [2, 3]. Angiogenesis plays a crucial role in growth, metastasis, and progression in RCC [4]. Therefore, blocking angiogenesis by vascular endothelial growth factor (VEGF) receptor-targeted therapies sorafenib (Nexavar, Bayer, Leverkusen, Germany), sunitinib (Sutent: Pfizer, New York, NY), pazopanib (Votrient: Novartis Oncology, East Hanover, NJ/before 2015: GlaxoSmithKline, Research Triangle Park, NC), tivozanib (Fotivda: EUSA Pharma, Hempstead, UK), cabozantinib (Cabometyx: Ipsen Pharma, Paris, France), or anti-VEGF antibodies such as bevacizumab (Avastin: Genentech, Roche, South San Francisco, CA; all other countries: Basel, Switzerland), has been the mainstay of therapy for more than a decade in patients with metastatic RCC (mRCC) [5]. The disease can also be treated with immunotherapy: interferon-alpha (INF- $\alpha 2 b$, IntronA: Merck \& Co., Whitehouse Station, NJ) and interleukin-2 (aldesleukin, Proleukin: Novartis Vaccines and Diagnostics, East Hanover, NJ) were first shown to be effective [6] followed by the checkpoint inhibitors nivolumab (Opdivo: Bristol Myers Squibb, New York, NY) and ipilimumab (Yervoy: Bristol Myers Squibb, New York, NY) [7]. Recently, immunotherapy combined with VEGF inhibitors has shown promising activity [8-11].

Dynamic contrast-enhanced computed tomography (DCE-CT) is a non-invasive functional imaging method that can assess angiogenesis. The method consists of repeated computed tomography (CT) scans of a tumour and can analyse changes in contrast enhancement in blood vessels and tissue over time. This enables an assessment of vascular support over the scanned tissue by performing a calculation of functional perfusion parameters such as blood flow (BF) and blood volume (BV), parameters which are closely correlated with angiogenesis
[12-14]. In patients with mRCC, high baseline BV has been associated with favourable baseline clinical factors, while low BV has been associated with unfavourable baseline clinical factors, and high baseline BV has been demonstrated as an independent favorable prognostic factor for survival outcome [15]. Furthermore, a decline in DCE-CT-identified parameters BV or BF during early treatment has shown correlation to favourable survival outcomes [16-18].

The degree of angiogenesis in a tumour can also be histologically characterised by the microvessel density (MVD) technique, where immunohistochemistry (IHC) staining is used to stain blood vessels [19]. Tumour MVD as a prognostic factor has been reported in several cancers, with high vascularity being associated with poorer patient outcomes in breast, ovarian, and prostate cancers [20-22]. In primary RCC, MVD as a prognostic factor for survival outcome has shown conflicting results [23-26], and there is limited knowledge about MVD as a prognostic factor in $\mathrm{mRCC}$. In patients with primary RCC [27, 28], assessment of angiogenesis using DCE-CT and MVD showed a positive correlation, whereas such correlation has not been assessed in patients with mRCC.

The primary aim of this study was to compare two different methods for assessing angiogenesis in the same tumour lesion at baseline in patients with mRCC: DCECT parameters BV and BF compared with MVD. The secondary aim was to assess the prognostic ability of BV, $\mathrm{BF}$, and MVD.

\section{Methods}

\section{Patients and treatment}

Patients with biopsy-verified mRCC were included in a prospective randomised phase II clinical trial, the Danish Renal Cancer Group Study-1 (DaRenCa-1), at Aarhus University Hospital, Denmark, from October 2009 to November 2014. Patients $(n=89)$ were randomised to receive subcutaneously administered interleukin 2 (IL-2) and interferon alpha (INF- $\alpha$ ) with or without intravenously administered bevacizumab ( $n=45$ and $n=44$, respectively). Functional imaging using DCE-CT and a corresponding core biopsy from the same tumour lesion was an integrated part of the study. All included patients were offered a biopsy, if safe and technically feasible at baseline.

Before entering the study, informed consent was obtained from all patients. The study was approved by the Danish Medicines Agency (journal no. 2612-4042), the Regional Ethics Committee (case no. 1-10-72-472-12), and the Danish Data Protection Agency (journal no. 2012-41-0897). Darenca-1 was registered at ClinicalTrials.gov (identifier NCT01274273). 
The clinical results from DaRenCa-1 [8], the prognostic impact on survival outcome of baseline DCE-CT identified BV [15], and the association between DCE-CT parameters and early treatment response [16-18] in this patient group have previously been published.

Electronic medical records were used to obtain baseline patient characteristics such as age, nephrectomy status, tumour histology, and the International Metastatic Renal Cell Carcinoma Database Consortium (IMDC) risk group factors [29].

\section{CT technique}

At baseline, DCE-CT and routine contrast-enhanced CT scans were performed using Philips Brilliance 64 or iCT 256 (Philips Healthcare, Best, The Netherlands). Firstly, a DCE-CT scan over a single target lesion was performed preceded by intravenous administration of 60 $\mathrm{mL}$ iodixanol (Visipaque, General Electric Healthcare, Princeton, NJ, USA) $270 \mathrm{mg} \mathrm{I} / \mathrm{mL}$ at $6 \mathrm{~mL} / \mathrm{s}$. The DCECT scans comprised of 2-s scan cycles (total $70 \mathrm{~s}$ ), median $100 \mathrm{kVp}$ (range, $80-120 \mathrm{kVp}$ ), median $z$-axis $8 \mathrm{~cm}$ (range, 4-13.5 cm), and median $100 \mathrm{mAs}$ (range, 100$210 \mathrm{mAs}$ ). The median CT dose index was $131.51 \mathrm{mGy}$ (range, 28.80-184.50 mGy). The median dose length product was $1,052.10 \mathrm{mGy} \times \mathrm{cm}$ (range, 434.80-27, $45.50 \mathrm{mGy} \times \mathrm{cm})$. Secondly, the patients remained supine for $10 \mathrm{~min}$ before performing a routine contrastenhanced CT of the thorax, abdomen, and pelvis preceded by administering intravenous iodixanol (Visipaque, GE Healthcare, Princeton, NJ) $270 \mathrm{mg} \mathrm{I} / \mathrm{mL}$ based on body weight (maximum $180 \mathrm{~mL}$ ) at $4 \mathrm{~mL} / \mathrm{s}$. In case of a previous minor reaction to iodixanol, administration of iohexol (Omnipaque, GE Healthcare, Princeton, NJ) $300 \mathrm{mg} \mathrm{I} / \mathrm{mL}$ was used. Contrast-enhanced CT was performed using $120 \mathrm{kVp}$ of peak voltage, $0.75 \mathrm{~s}$ of rotation time, a pitch of 0.925 , a collimation of 64 or $128 \times 0.625$ $\mathrm{mm}$, and attenuation-based current modulation. Prior to the administration of intravenous contrast agent media, all patients were given 1-L saline solution intravenously to ensure hydration.

Routine contrast-enhanced CT scans were performed every third month until progressive disease and assessed according to the criteria laid out in Response Evaluation Criteria in Solid Tumors, version 1.1 (RECIST v.1.1) [30, 31] by experienced radiologists and used for clinical purpose, whereas DCE-CT scans were used exclusively for research purpose.

\section{Image analysis}

DCE-CT data were analysed in four dimensions using the Advanced Perfusion and Permeability Application (APPA) (Philips, Healthcare, Best, The Netherlands).

After loading the dynamic data in the APPA software program, a non-rigid registration used for motion correction and spatial filtration was performed. The dynamic data were processed and analysed at arterial peak enhancement. DCE-CT parameters BV deconvolution $\left(\mathrm{BV}_{\text {deconv }}, \mathrm{mL} \times 100 \mathrm{~g}^{-1}\right)$ and $\mathrm{BF}$ deconvolution $\left(\mathrm{BF}_{\mathrm{de}-}\right.$ conv, $\mathrm{mL} \times 100 \mathrm{~g}^{-1} / \mathrm{min}$ ) were calculated using the deconvolution method; BV Patlak (BV Patlak, $\mathrm{mL} \times 100 \mathrm{~g}^{-1}$ ) was calculated using the two-compartment method [13]. The corresponding BV and BF series were displayed together with the morphologic series at arterial peak enhancement.

DCE-CT data were loaded into Intellispace 6.0 Multimodality Tumor Tracking, (Philips, Healthcare, Best, The Netherlands), where the entire target lesion was segmented on the morphologic series at arterial peak enhancement using a three-dimensional semiautomatic sculpt tool and was defined as the volume of interest (VOI). A blinded radiologist with 2 years of training in DCE-CT performed all the analyses (A.D.N.). An excellent interobserver correlation in the analyses of functional imaging CT data in this patient group has previously been found [18].

Dynamic data were loaded and analysed in MATLAB (v. R2015b, MathWorks Inc., Natick, MA, USA) based on the delineated VOI at arterial peak enhancement using an in-house software.

The median values for the DCE-CT parameters were calculated using histogram analysis. We used the median values of $\mathrm{BV}_{\text {deconv }}, \mathrm{BV}_{\text {Patlak }}$, and $\mathrm{BF}_{\text {deconv, }}$, as these three parameters have shown the best correlation with survival outcome in patients with mRCC [18]. In addition, the contrast-enhanced tissue density measured as Hounsfield units (HU) was assessed for each target lesion at arterial peak enhancement.

\section{Biopsies}

According to prespecified protocol criteria, experienced radiologists (H.H.T.M. or J.M.) defined either a primary tumour or metastasis as a target lesion at baseline, suitable for core biopsy and functional imaging using CT [32].

All core biopsies were performed by an experienced radiologist under ultrasound guidance either the same day as the DCE-CT and contrast-enhanced CT scan or the subsequent day. As a standard, two core biopsy samples were taken from each target lesion using an 18-G automated core biopsy needle. As a default, the same target lesion was used for DCE-CT and core biopsy, i.e., matched target lesions. However, this was not always technically possible for all target lesions. Out of the 89 included patients, a total of 43 patients had a biopsy performed at baseline. Unmatched biopsies $(n=9)$ and biopsies without representative tumour specimen ( $n$ $=9$ ) were excluded from the study. A total of 25 
Table 1 Target lesion localisation

\begin{tabular}{llll}
\hline & $\begin{array}{l}\text { All patients } \\
(\boldsymbol{n}=\mathbf{2 5})\end{array}$ & Patients with synchronous metastases $(\boldsymbol{n}=\mathbf{1 9 )}$ & Patients with metachronous metastases $(\boldsymbol{n}=\mathbf{6})$ \\
\hline Primary kidney tumour & 4 & 4 & - \\
Liver & 4 & 2 & 2 \\
Lung/pleura & 4 & 4 & - \\
Lymph node & 2 & 2 & - \\
Kidney (metastases) & 2 & 1 & 1 \\
Retroperitoneum & 4 & 2 & 2 \\
Bone (soft tissue component) & 5 & 4 & 1 \\
\hline
\end{tabular}

patients with a matched target lesion were included in the final analysis (Table 1).

\section{IHC staining and MVD}

The core biopsy specimens were fixed with $10 \%$ formaldehyde and paraffin-embedded. Each paraffin block was cut into $3-\mu$ m-thick serial slices and mounted on glass slides. From each paraffin block, one slide was stained with hematoxylin and eosin (Ventana HE 600, Ventana Medical Systems, Tucson, AZ, USA) to identify tumour regions, and one slide was stained with monoclonal mouse antibody CD34 (QBEnd-10; Ventana, as above) by Benchmark XT (Ventana) to assess MVD. CD34 was chosen to quantify the vessels in the tumour, as this method has been shown to be specific and reproducible for this purpose [33, 34]. Standard settings and reagent kits were used in deparaffinisation, rehydration, antigen retrieval, and endogenous peroxidase blocking. CD34 was visualised by OptiView 3,3'-Diaminobenzidine IHC Detection Kit (Ventana, as above). Using Mayer's hematoxylin and bluing reagent, all slides were counterstained. Both slides were digitalised at $20 x$ by NanoZoomer 2.0HT (Hamamatsu Photonics K.K., Hamamatsu City, Japan) and superimposed to form a virtual stain in the Visiopharm Integrator System 2019.02.1.6005 (Visiopharm A/S, Hørsholm, Denmark).

An experienced uropathologist (C.S.) with 10 years of pathology training manually outlined tumour regions and necrosis regions on the digitised HE slides in Visiopharm; these outlines were automatically transferred to the CD34 slides within the virtual slide. Based on a threshold of the CD34 staining colour, marked by a colour deconvolution step, an automated quantification of CD34 was obtained. To exclude inaccuracies of the automated detection, all analyses were also reviewed manually by P.S.N., with 10 years of training in digital pathology. MVD was assessed using the CD34-index in both the tumour outline (T), defined as the CD34-index ${ }_{\mathrm{T}}$, and in the combined tumour and necrosis outline (TN), defined as CD34index $_{\mathrm{TN}}$, respectively, and was calculated using the following equation:

$$
\frac{\text { CD34 positive } \mathrm{T} \text { or } \mathrm{TN} \text { area }}{\text { Total T or TN area }} \times 100 \%
$$

\section{Statistical analysis}

Progression-free survival (PFS) was defined as the period between study inclusion and RECIST v1.1-defined progression/cancer-related death. The period between inclusion and death was defined as the overall survival (OS). Kaplan-Meier survival curves and log-rank tests were used to test the association between baseline CD34-index as a categorical variable, using the median value as a cutoff, (> versus $\leq$ median) and survival outcome. The association between CD34-index as a continuous variable and survival outcome was tested using Cox regression analysis. The association between baseline CD34-index and baseline clinical factors, age, gender, and treatment group, was tested using $X^{2}$ test or Fischer exact test, when applicable. Synchronous metastatic disease is defined as presence of metastases $\leq 3$ months of initial cancer diagnosis, metachronous metastatic disease as presence of metastases $>3$ months of initial diagnosis.

The median follow-up time in alive patients was assessed using the reverse Kaplan-Meier survival method. The association between baseline clinical factors in this present study $(n=25)$ was compared to baseline clinical factors in the previously published study $(n=105)$ [15] using $X^{2}$ or Fischer exact test, when applicable.

DCE-CT parameters and CD34-index had a clear nonGaussian distribution assessed visually on probability histograms. Therefore, non-parametric Spearman's rank correlation coefficient, rho, was used to test the correlation between DCE-CT parameters or $\mathrm{HU}$ and the CD34-index in the tumour outline and the combination of tumour and necrosis outline, respectively; $p$-values < 0.05 were regarded as statistically significant. All statistical tests were two-sided. 
All statistical analyses were performed using IBM SPSS Statistics for Windows (Version 27.0, IBM Corp., Armonk, NY, USA).

\section{Results}

\section{Patient characteristics}

At baseline, 25 patients had analysable scans and tissue (Table 1). All patients had clear cell tumour histology. The median age was 57.2 years (range 37.5-66.4), 18 (72\%) patients were male, and 19 (76\%) patients had synchronous metastatic disease. According to the IMDC classification, two (8\%) patients had favourable, 18 (72\%) patients had intermediate, and five $(20 \%)$ patients had poor prognosis. A total of $13(52 \%)$ patients were treated with IL-2 and IFN- $\alpha$, and $12(48 \%)$ patients were treated with IL-2, IFN- $\alpha$, and bevacizumab. The median volume of the target lesions was $44.9 \mathrm{~cm}^{3}$ (range 4.79427.45). The median follow-up time in alive patients was 112.3 months, the median PFS was 6.3 months (range 0.5-118.6), and the median OS was 27.8 months (range 0.9-125.0).

To control for imbalances in established prognostic factors, we compared baseline patient characteristics in the present cohort $(n=25)$ with the previously published cohort $(n=105)$ [15]. We found no difference in IMDC risk groups $(p=0.188)$, sex $(p=0.893)$, treatment $(p=0.122)$, age $(p=0.371)$, synchronous versus. metachronous disease $(p=0.083)$, or target lesion volume ( $p$ $=0.589$ ).

\section{MVD and DCE-CT parameters}

The CD34-index ${ }_{\mathrm{T}}$ and CD34-index $\mathrm{x}_{\mathrm{TN}}$ at baseline were 6.76\% (range 0.78-19.23) and 6.15\% (range 0.78-19.23), respectively. Median $\mathrm{BV}_{\text {deconv }}$ was $29.00 \mathrm{~mL} \times 100 \mathrm{~g}^{-1}$ (range 11.41-70.03), BV Patlak was $20.45 \mathrm{~mL} \times 100 \mathrm{~g}^{-1}$ (range 3.69-68.64), $\mathrm{BF}_{\text {deconv }}$ was $159.88 \mathrm{~mL} \times 100 \mathrm{~g}^{-1} /$ min (range 52.33-346.24), and HU was 89.00 (range 50.00-231.00).

The histogram width (i.e., the difference between the minimum and maximum values of the histograms) at baseline for $\mathrm{BV}_{\text {deconv }}$ was $90.69 \mathrm{~mL} \times 100 \mathrm{~g}^{-1}$ (range 24.38-618.21), for $B V_{\text {Patlak }}$ was $68.28 \mathrm{~mL} \times 100 \mathrm{~g}^{-1}$ (range 23.83-381.85), for $\mathrm{BF}_{\text {deconv }}$ was $511.42 \mathrm{~mL} \times 100$ $\mathrm{g}^{-1} / \mathrm{min}$ (range 86.67-3,640.46), and for the tissue density was $366 \mathrm{HU}$ (range 75.00-1,882.00) (Fig. 1).

An example of CE-CT, DCE-CT, and CD34 IHC staining from the same target lesion in a representative patient is illustrated in Fig. 2.

\section{MVD and baseline characteristics}

No statistically significant association was found between CD34-index $_{\mathrm{TN}}$ and age $(p=0.543)$, sex $(p=0.748)$, treatment group $(p=0.848)$, IMDC prognostic group ( $p$
$=0.152)$, target lesion volume $(p=0.434)$, or synchronous versus. metachronous metastatic disease $(p=0.378)$. Similarly, no association was found between CD34index $\mathrm{T}_{\mathrm{T}}$ and baseline patient characteristics (Table 2).

\section{MVD, DCE-CT, and survival outcome}

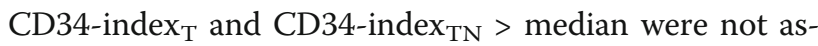
sociated with survival outcome, with similar PFS (6.7 versus 5.6 months; $p=0.441$ and $p=0.854$, respectively) and near-identical OS (27.8 versus 27.4 months; $p=$ 0.987 and $p=0.528$, respectively). DCE-CT parameters $\mathrm{BV}_{\text {deconv }}, \mathrm{BV}_{\text {Patlak, }}$, and $\mathrm{BF}_{\text {deconv }}>$ median were all associated with favourable OS (43.2 versus 14.6 months, $p=$ 0.002 ; 31.6 versus $20.2, p=0.015$; and 31.6 versus 24.5 months, $p=0.019$, respectively), but not with PFS (5.3 versus 6.3 months, $p=0.411 ; 6.7$ versus 5.3 months, $p=$ 0.317 ; and 5.3 versus $6.3, p=0.531$, respectively) (Fig. 3 ).

Assessing baseline CD34-index ${ }_{\mathrm{T}}$ and CD34-index $\mathrm{TNN}_{\mathrm{TN}}$ as a continuous variable also showed no correlation with PFS (hazard ratio (HR) 0.99, 95\% confidence interval (CI) $0.93-1.06, p=0.824$ and HR 1.00 , 95\% CI $0.94-$ 1.07, $p=0.905$ ) or OS (HR 1.00, 95\% CI 0.93, 1.08, $p=$ 0.930 and HR 1.01 ; $95 \%$ CI $0.95,1.08, p=0.684$ ), respectively. Adjusting baseline CD34-index ${ }_{\mathrm{T}}$ and CD34index $_{\mathrm{TN}}$ for treatment group, there still was no correlation with PFS (HR 1.03, 95\% CI 0.96-1.11, $p=0.404$ and HR 1.04, 95\% CI $0.97-1.11, p=0.288)$ and OS (HR 1.00, 95\% CI 0.93-1.08, $p=0.936$ and HR 1.02, 95\% CI $0.95-1.09, p=0.677$ ), respectively.

\section{Correlation between MVD and DCE-CT parameters}

No statistically significant correlation was found between CD34-index $\mathrm{T}_{\mathrm{T}}$ or CD34-index $\mathrm{TN}_{\mathrm{TN}}$ and DCE-CT-derived parameters BV and BF at baseline (Table 3 and Fig. 4). This lack of correlation remained when stratifying patients for synchronous versus metachronous mRCC (Table 3). Furthermore, there was no correlation between $\mathrm{HU}$ and CD34-index $\mathrm{T}_{\mathrm{T}}$ or CD34-index ${ }_{\mathrm{TN}}$, respectively (Table 3 and Fig. 4).

\section{Discussion}

To our knowledge, this study is the first to assess two different methods in the evaluation of angiogenesis in patients with mRCC. At baseline from the same tumour lesion, a core biopsy was obtained to assess MVD by immunohistochemistry, in parallel with a DCE-CT scan to calculate BV and BF. We identified $\mathrm{BV}$ and $\mathrm{BF}$ to have strong prognostic ability; patients with high BV or BF had favourable survival. In contrast, MVD did not show prognostic potential, and moreover, MVD did not correlate to baseline patient characteristics, to DCE-CT parameters $\mathrm{BV}$ or $\mathrm{BF}$, or to $\mathrm{HU}$. Despite only 25 patients had evaluable scans and tumours, this study 

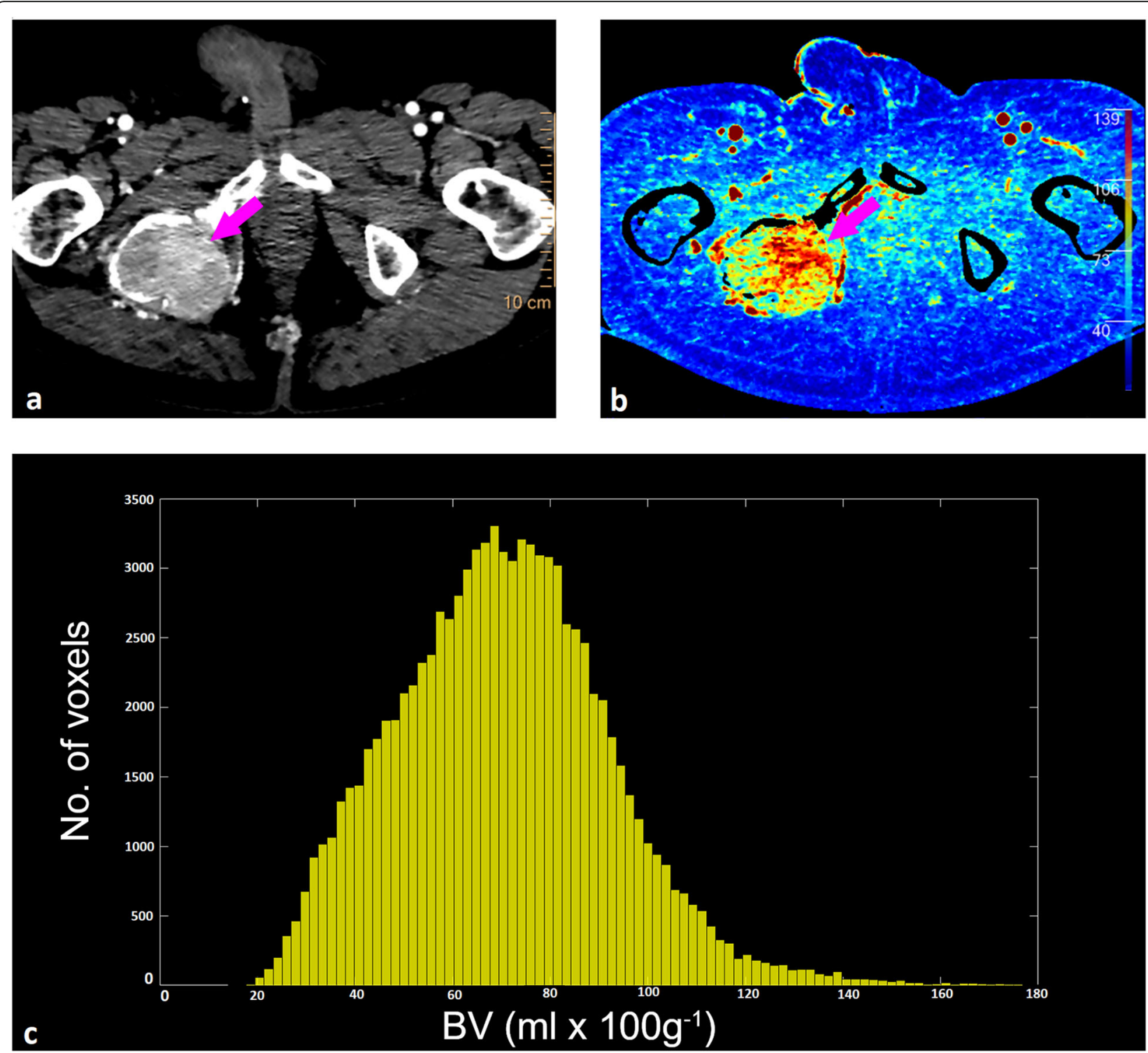

Fig. 1 A heterogeneous metastasis in the right iliac bone (purple arrow) at baseline presented on (a) CE- $C T$, (b) BV deconv map, and (c) the corresponding $\mathrm{BV}_{\text {deconv }}$ histogram. The histogram depicts a large BV range, illustrating the intratumoural heterogeneity. $B V_{\text {deconv }} B$ Blood volume (deconvolution), CE-CT Contrast-enhanced computed tomography, No. Number

represents the largest cohort of patients with metastatic renal cell carcinoma examined to date with an integrated prospectively obtained functional imaging $\mathrm{CT}$ and corresponding biopsy design [8]; all analyses were preplanned per protocol.

No correlations between DCE-CT parameters BV and $\mathrm{BF}$ and MVD were found regardless of assessing the CD34-index in isolated tumours or tumours including necrosis. This lack of correlation remained after stratification of patients into those with synchronous mRCC and metachronous mRCC. Although, no adjustments for multiple testing were performed in this study, there was no statistically significant correlation, which further highlighted the lack of correlation. Furthermore, baseline MVD was not associated with baseline patient characteristics, $\mathrm{HU}$, or with survival outcome independent of treatment group, thus indicating that baseline MVD does not have prognostic impact in mRCC. However, further research evaluating this matter in a larger cohort is encouraged, including evaluating the correlation between DCE-CT parameters and MVD during ongoing oncologic treatment.

Two previous studies have found a positive correlation between DCE-CT-identified parameters and MVD assessed by CD34 IHC in primary RCC undergoing nephrectomy [27, 28]. Chen et al. [27] found a positive 

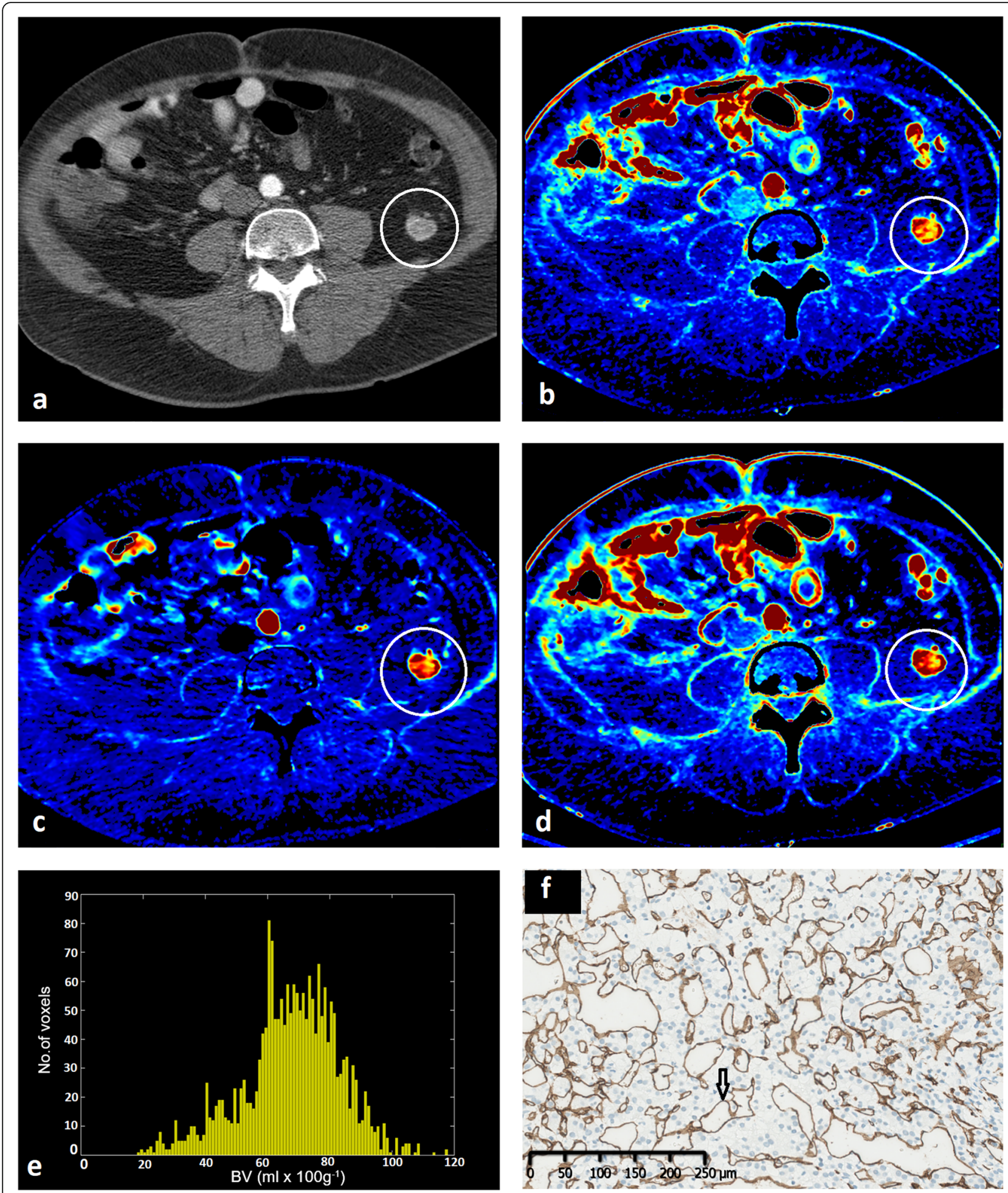

Fig. 2 A retroperitoneal metastasis marked with a white circle depicted on (a) a contrast-enhanced $C T$ at peak arterial enhancement with the corresponding functional imaging colour maps, (b) $\mathrm{BV}_{\text {deconv, }}$ (c) $\mathrm{BV}_{\text {Patlak, }_{\text {, }} \text { and }}(\mathbf{d}) \mathrm{BF}_{\text {deconv. }} \mathrm{BV}_{\text {deconv }}$ histogram for the entire target lesion at baseline is shown in $\mathbf{e}$, while $\mathbf{f}$ represents the CD34 immunostaining from the same retroperitoneal metastasis with microvessels (arrow) stained brown. $B V_{\text {deconv }}$ Blood volume (deconvolution), $B V_{\text {Patlak }}$ Blood volume (Patlak), $B F_{\text {deconv }}$ Blood flow (deconvolution) 
Table 2 Baseline patient characteristics

\begin{tabular}{|c|c|c|c|}
\hline & \multicolumn{3}{|l|}{ CD34-index $x_{T}$} \\
\hline & Above median (>), $n(\%)$ & Below median $(\leq), \boldsymbol{n}(\%)$ & $p$-value \\
\hline Total & 12 & 13 & \\
\hline Age (years) & & & 0.543 \\
\hline > Median (57.2) & $5(41.7)$ & $7(58.3)$ & \\
\hline$\leq$ Median (57.2) & $7(53.8)$ & $6(46.2)$ & \\
\hline Sex & & & 0.225 \\
\hline Male & $10(55.6)$ & $8(44.4)$ & \\
\hline Female & $2(28.6)$ & $5(71.4)$ & \\
\hline Treatment group & & & 0.848 \\
\hline IL2/ IFN-a & $6(46.2)$ & $7(53.8)$ & \\
\hline IL2/INF-a/bevacizumab & $6(50.0)$ & $6(50.0)$ & \\
\hline IMDC category & & & 0.152 \\
\hline Favourable & $2(100.0)$ & - & \\
\hline Intermediate & $9(50.0)$ & $9(50.0)$ & \\
\hline Poor & $1(20.0)$ & $4(80.0)$ & \\
\hline Target lesion volume $\left(\mathrm{cm}^{3}\right)$ & & & 0.848 \\
\hline > Median (44.9) & $6(46.2)$ & $7(53.8)$ & \\
\hline$\leq$ Median (44.9) & $6(50.0)$ & $6(50.0)$ & \\
\hline Synchronous metastases & & & 0.378 \\
\hline Yes & $8(42.1)$ & $11(57.9)$ & \\
\hline No & $4(66.7)$ & $2(33.3)$ & \\
\hline
\end{tabular}

correlation between MVD and DCE-CT-identified BV, $\mathrm{BF}$, and permeability surface area product in 77 patients that had undergone surgical resection for primary RCC. In that study, the DCE-CT parameters were assessed using one circular ROI on a 5-mm tumour section, and MVD was assessed in the surgical specimen sampled at the level corresponding to the DCE-CT assessment. Wang et al. also found a positive correlation between DCE-CT parameters and MVD in 24 patients with primary RCC undergoing surgical resection [28]. In that study, DCE-CT scans were assessed using either one or two circular ROIs, while MVD was assessed from the surgical specimen sampled corresponding to the ROIs selected at DCE-CT [28]. Employing this method, Chen et al. [27] and Wang et al. [28] assessed the DCE-CT parameters and MVD in the exactly same region of the tumour and found a close correlation between BV or BF and MVD. In contrast, the DCE-CT-identified parameters in our study were assessed using histogram analysis of the entire target lesion, mostly metastatic lesions, using a VOI, whereas MVD was assessed from a single core biopsy; this could explain the difference in our results compared to the results of Wang et al. [28] and Chen et al. [27]. Consistent with our findings, a study by Puerto-Nevado et al. [35] in patients with mRCC found no prognostic impact of MVD assessed at metastatic sites in 23 patients treated with sunitinib.

Recently, the evolutionary trajectories of kidney cancer were described in more detail by the TRACERx Renal study, and the authors identified large intratumoural heterogeneity in mRCC [36]. The results from our study indicated that angiogenesis assessed by a single core biopsy from a target lesion may not reflect the angiogenesis of the entire target lesion and support the findings of intratumoral heterogeneity in mRCC [36]. The great width of the histograms (i.e., the large difference between the minimum and maximum values of the histogram) in our assessments also reflects the intratumoural heterogeneity. Furthermore, heterogeneity between patients with synchronous and metachronous mRCC has been shown [37, 38]. However, in our study, we found no difference between baseline MVD in patients with synchronous versus metachronous $\mathrm{mRCC}$, and the lack of correlation between MVD and DCE-CT parameters remained after analysing patients with synchronous and metachronous mRCC separately.

We have previously established that the DCE-CT parameter BV was an independent prognostic factor that added to the prognostic accuracy of IMDC [15-18]. 


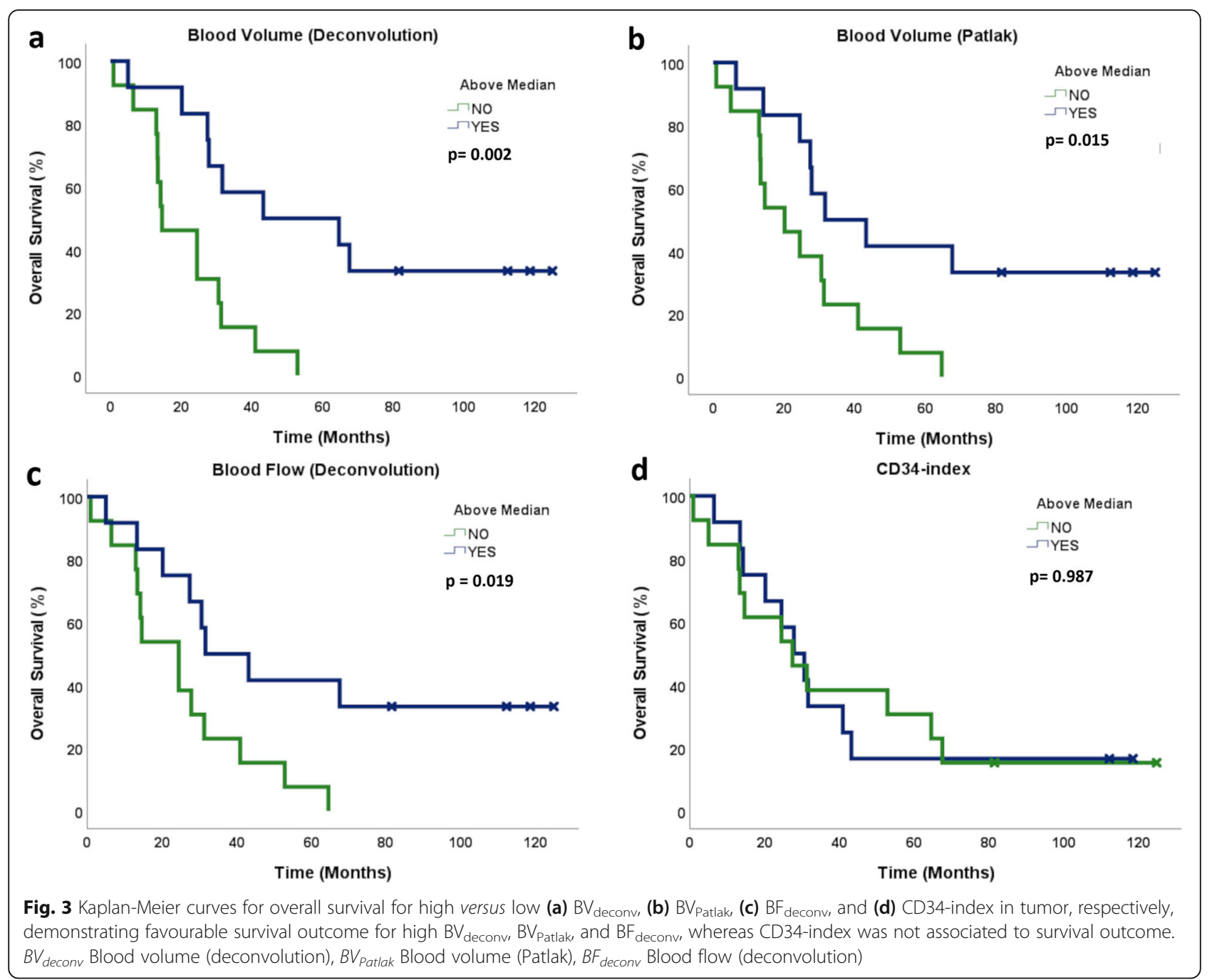

Analogous to our previous findings, we were able to identify the same positive association between overall survival and baseline $\mathrm{BV}_{\text {deconv, }} \mathrm{BV}_{\text {Patlak }}$, or $\mathrm{BF}_{\text {deconv }}$ in only 25 patients in the present study. This finding highlights the robustness and strength of DCE-CT parameters $\mathrm{BV}$ and $\mathrm{BF}$ as prognostic factors. Furthermore, we found no difference in baseline patient characteristics between patients from this study $(n=25)$ and the previous study $(n=105)$, thereby excluding the risk of sampling bias.

Assessment of angiogenesis using MVD has several disadvantages: it is an invasive procedure that is associated with greater discomfort and risk of procedure complications, it does not allow repeated measurements, and

Table 3 Correlations between DCE-CT parameters, HU, and CD34-index at baseline

\begin{tabular}{|c|c|c|c|c|c|c|}
\hline & \multicolumn{2}{|c|}{$\begin{array}{l}\text { All patients }(n=25) \\
\text { SCC }(p \text {-value) }\end{array}$} & \multicolumn{2}{|c|}{$\begin{array}{l}\text { Synchronous mRCC }(n=19) \\
\text { SCC ( } p \text {-value) }\end{array}$} & \multicolumn{2}{|c|}{$\begin{array}{l}\text { Metachronous mRCC }(n=6) \\
\text { SCC ( } p \text {-value) }\end{array}$} \\
\hline & CD34-index ${ }_{T}$ & $\overline{\text { CD34-index }}$ & CD34-index ${ }_{T}$ & $\overline{\text { CD34-index }}$ & CD34-index & CD34-index ${ }_{\mathrm{TN}}$ \\
\hline $\mathrm{BV}_{\text {deconv }}$ & $0.23(0.275)$ & $0.21(0.317)$ & $0.20(0.416)$ & $0.19(0.429)$ & $0.14(0.787)$ & $0.14(0.787)$ \\
\hline$B V_{\text {Patlak }}$ & $0.20(0.351)$ & $0.17(0.417)$ & $0.23(0.336)$ & $0.22(0.367)$ & $0.09(0.872)$ & $0.09(0.872)$ \\
\hline $\mathrm{BF}_{\text {deconv }}$ & $0.26(0.207)$ & $0.22(0.289)$ & $0.27(0.273)$ & $0.23(0.344)$ & $0.14(0.787)$ & $0.14(0.787)$ \\
\hline $\mathrm{HU}$ & $0.20(0.351)$ & $0.22(0.285)$ & $0.12(0.624)$ & $0.18(0.466)$ & $0.31(0.544)$ & $0.31(0.544)$ \\
\hline
\end{tabular}

$B V_{\text {deconv }}$ Blood volume (deconvolution), $B V_{\text {Patlak }}$ Blood volume (Patlak), $B F_{\text {deconv }}$ Blood flow (deconvolution), $C D 34-i n d e x_{T N} C D 34$-index in the tumour necrosis outline, CD34-index $x_{T}$ CD34-index in the tumour outline, DCE-CT Dynamic contrast-enhanced computed tomography, HU Hounsfield units, mRCC Metastatic renal cell carcinoma, SCC Spearman correlation coefficient (rho) 

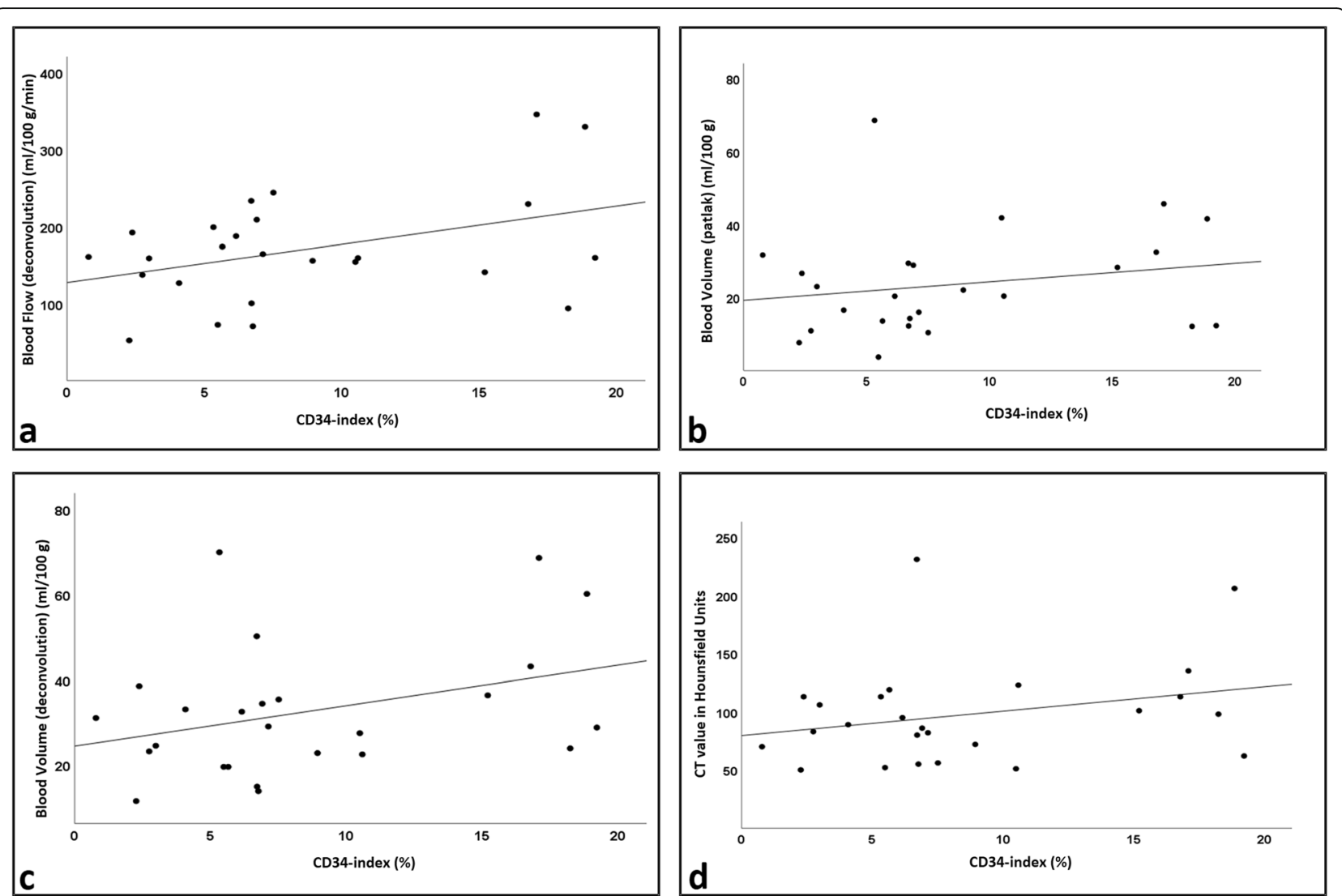

Fig. 4 Plots illustrating no correlation between CD34-index in tumor outlines and (a) $B F_{\text {deconv }}$ (b) $B V_{\text {Patlak, }}$ (c) $B V_{\text {deconv, }}$ and (d) $H U$, respectively at baseline. $B V_{\text {deconv }}$ Blood volume (deconvolution), $B V_{\text {Patlak }}$ Blood volume (Patlak), BF deconv Blood flow (deconvolution), HU Hounsfield units

it does not take account for the intratumoural heterogeneity [27, 36]. However, assessment of angiogenesis can be performed noninvasively using functional imaging DCE-CT scan method; by histogram analysis of the entire tumour lesion, this method takes account for intratumoural heterogeneity [12-14]. In contrast, a single core biopsy only provides information from a small fraction of the tumour [36]. The non-invasive DCE-CT method may be more suitable in the assessment of angiogenesis in patients with $\mathrm{mRCC}$.

The study was limited by the low number of patients. A limitation to DCE-CT is motion artefacts due to the repeated scans. However, the motion artefacts were minor and could be corrected using the non-rigid registration feature in the APPA software program, which resulted in no patients being excluded due to motion artefacts. Another limitation to the DCE-CT technique is the high radiation dose. However, due to the reduced life expectancy in $\mathrm{mRCC}$, the risk of radiation-induced cancer is considered to be relatively low.

In conclusion, DCE-CT parameters BV and BF had a prognostic impact and may better reflect angiogenesis in mRCC. However, validation in a larger study is encouraged.

\section{Abbreviations}

APPA: Advanced perfusion and permeability application; BF: Blood flow; $\mathrm{BV}$ : Blood volume; $\mathrm{BF}_{\text {deconv: }} \mathrm{BF}$ deconvolution; Cl: Confidence interval; CT: Computed tomography; DaRenCa-1: Danish Renal Cancer Group Study-1; DCE-CT: Dynamic contrast-enhanced CT; HR: Hazard ratio;

IHC: Immunohistochemistry; IL-2: Interleukin 2; IMDC: International Metastatic Renal Cell Carcinoma Database Consortium; INF-a: Interferon alpha; mRCC: Metastatic renal cell carcinoma; MVD: Microvessel density; OS: Overall survival; PFS: Progression-free survival; RECIST v1.1: Response Evaluation Criteria in Solid Tumors, version 1.1; T: Tumour outline; TN: Tumour and Necrosis outline; VEGF: Vascular endothelial growth factor; VOI: Volume of interest

\section{Acknowledgements}

We wish to acknowledge Philips Healthcare, who provided the software used for the imaging analysis, and Roche and Novartis, who supported the clinical part of the study financially, but were not involved in the imaging analysis. We also wish to acknowledge Ipsen, the Health Research

Foundation of Central Denmark, the Maersk Foundation, and the Memorial Foundation of Eva and Henry Fraenkel for supporting the study financially.

\section{Authors' contributions}

ADN: conceptualisation, data curation, formal analysis, investigation, methodology, project administration, original craft, writing, review, and editing. FR: conceptualisation, data curation, formal analysis, investigation, methodology, project administration, supervision, writing, review, and editing. PSN: data curation, formal analysis, investigation, methodology, software, writing, review, and editing. CS: data curation, formal analysis, investigation, methodology, writing, review, and editing. KT: data curation, formal analysis, investigation, methodology, software, writing, review, and editing. JM: conceptualisation, data curation, formal analysis, investigation, 
methodology, supervision, writing, review, and editing. HTM: data curation, methodology, writing, review, and editing. FD: conceptualization, data curation, formal analysis, investigation, methodology, funding acquisition, project administration, supervision, writing, review, and editing. All authors have approved the final version to be published and are accountable for all aspects of the work in ensuring that questions related to the accuracy or integrity of any part of the work are appropriately investigated and resolved.

\section{Funding}

Philips Healthcare provided the software used for the imaging analysis. Roche and Novartis supported the clinical part of the study financially. Ipsen, the Health Research Foundation of Central Denmark, the Maersk Foundation, and the Memorial Foundation of Eva and Henry Fraenkel supported the study financially.

\section{Availability of data and materials}

The datasets generated and/or analyzed during the current study are not publicly available due to further analysis of data for upcoming publications, but are available from the corresponding author on reasonable request.

\section{Declarations}

\section{Ethics approval and consent to participate}

Trial Registration: Darenca-1 study was approved by the Danish Ethics Committee (URL: https://www.rm.dk/sundhed/faginfo/forskning/devidenskabsetiske-komiteer/) (case no. 1-10-72-472-12) on the 23rd of June 2009, the Danish Data Protection Agency (URL: https://www.rm.dk/sundhed/ faginfo/forskning/Forskningsprojekter/) (journal no. 2012-41-0897) on the 25th of May 2009, and the Danish Medicines Agency (URL: https:// laegemiddelstyrelsen.dk/) (journal no. 2612-4042) on the 1st of July 2009; the study was registered at ClinicalTrials.gov (identifier NCT01274273). Approval by the Regional Ethics Committee, the Danish Medicines Agency, and The Danish Data Protection Agency was granted, and written informed consent was obtained before inclusion started.

\section{Consent for publication}

Not applicable.

\section{Competing interests}

A. Drljevic-Nielsen reports receiving research grants from Ipsen, the Maersk Foundation, and the Health Research Foundation of Central Denmark Region, and conference travels from Pfizer and Ipsen. F. Donskov reports receiving research grants from Ipsen, Pfizer, MSD, and the Health Research Foundation of Central Denmark Region. JR. Mains reports receiving research grants from the Memorial Foundation of Eva and Henry Fraenkel and the Health Research Foundation of Central Denmark Region and conference travels from Pfizer. No potential conflicts of interest were disclosed by C. Stilling, P. Switten Nielsen, F. Rasmussen, and K. Thorup.

\section{Author details}

'Department of Oncology, Aarhus University Hospital (AUH), Palle Juul-Jensens Blvd. 99, 8200 Aarhus N, Denmark. ²Department of Radiology, Aarhus University Hospital (AUH), Palle Juul-Jensens Blvd. 99, 8200 Aarhus N, Denmark. ${ }^{3}$ Department of Pathology, Aarhus University Hospital (AUH), Palle Juul-Jensen Blvd. 99, 8200 Aarhus N, Denmark.

\section{Received: 12 March 2021 Accepted: 1 July 2021}

Published online: 30 July 2021

\section{References}

1. Chow WH, Dong LM, Devesa SS (2010) Epidemiology and risk factors for kidney cancer. Nat Rev Urol 7:245-257 https://doi.org/210.1038/nrurol.2010.1 046

2. Nickerson $M L$, Jaeger $E$, Shi $Y$, et al (2008) Improved identification of von Hippel-Lindau gene alterations in clear cell renal tumors. Clin Cancer Res 14: 4726-4734 https://doi.org/1158/1078-0432.CCR-4707-4921. https://doi.org/1 0.1158/1078-0432.CCR-07-4921

3. Baldewijns MM, Thijssen VL, Van den Eynden GG et al (2007) High-grade clear cell renal cell carcinoma has a higher angiogenic activity than lowgrade renal cell carcinoma based on histomorphological quantification and
qRT-PCR mRNA expression profile. Br J Cancer 96:1888-1895 https://doi. org/1810.1038/sj.bjc.6603796

4. Jilaveanu LB, Puligandla M, Weiss SA, et al (2018) Tumor microvessel density as a prognostic marker in high-risk renal cell carcinoma patients treated on ECOG-ACRIN E2805. Clin Cancer Res 24:217-223. https://doi.org/10.1158/1 078-0432.Ccr-1117-1555

5. Canino C, Perrone L, Bosco E, et al (2019) Targeting angiogenesis in metastatic renal cell carcinoma. Expert Rev Anticancer Ther 19:245-257 https://doi.org/210.1080/14737140.14732019.11574574

6. Bukowski RM (2000) Cytokine combinations: therapeutic use in patients with advanced renal cell carcinoma. Semin Oncol 27:204-212

7. Motzer RJ, Escudier B, McDermott DF et al (2020) Survival outcomes and independent response assessment with nivolumab plus ipilimumab versus sunitinib in patients with advanced renal cell carcinoma: 42-month followup of a randomized phase 3 clinical trial. J Immunother Cancer 8:e000891 https://doi.org/000810.001136/jitc-002020-000891

8. Donskov F, Jensen NV, Smidt-Hansen T et al (2018) A randomized phase II trial of interleukin-2 and interferon-alpha plus bevacizumab versus interleukin-2 and interferon-alpha in metastatic renal-cell carcinoma (mRCC): results from the Danish Renal Cancer Group (DaRenCa) study-1. Acta Oncol 57:589-594 https://doi.org/510.1080/02 84186x.0282018.1433324

9. Rini BI, Plimack ER, Stus V, et al (2019) Pembrolizumab plus axitinib versus sunitinib for advanced renal-cell carcinoma. N Engl J Med 380:1116-1127 https://doi.org/1110.1056/NEJMoa1816714

10. Motzer RJ, Penkov K, Haanen J, et al (2019) Avelumab plus axitinib versus sunitinib for advanced renal-cell carcinoma. N Engl J Med 380:1103-1115 https://doi.org/1110.1056/NEJMoa1816047

11. Motzer R, Alekseev B, Rha S-Y et al (2021) Lenvatinib plus pembrolizumab or everolimus for advanced renal cell carcinoma. N Engl J Med 384:1289-1300

12. Miles KA, Lee TY, Goh V et al (2012) Current status and guidelines for the assessment of tumour vascular support with dynamic contrast-enhanced computed tomography. Eur Radiol 22:1430-1441 https://doi.org/1410.1007/ s00330-00012-02379-00334

13. Miles KA, Griffiths MR (2003) Perfusion CT: a worthwhile enhancement? Br J Radiol 76:220-231 http://doi.org/210.1259/bjr/13564625

14. Miles KA (2006) Perfusion imaging with computed tomography: brain and beyond. Eur Radiol 16:M37-M43. https://doi.org/10.1007/s10406-10006-101 94-10401

15. Drljevic-Nielsen A, Rasmussen F, Mains JR, Thorup K, Donskov F (2020) Baseline blood volume identified by dynamic contrast-enhanced computed tomography as a new independent prognostic factor in metastatic renal cell carcinoma. Transl Oncol 13:100829 https://doi.org/100810.101016/j.tra non.102020.100829

16. Mains JR, Donskov F, Pedersen EM, Madsen HHT, Rasmussen F (2014) Dynamic contrast-enhanced computed tomography as a potential biomarker in patients with metastatic renal cell carcinoma: preliminary results from the Danish Renal Cancer Group Study-1. Invest Radiol 49:601607 https://doi.org/610.1097/rli.0000000000000058

17. Mains JR, Donskov F, Pedersen EM, Madsen HHT, Rasmussen F (2017) Dynamic contrast-enhanced computed tomography-derived blood volume and blood flow correlate with patient outcome in metastatic renal cell carcinoma. Invest Radiol 52:103-110 https://doi.org/110.1097/RLI. 0000000000000315

18. Mains JR, Donskov F, Pedersen EM et al (2018) Use of patient outcome endpoints to identify the best functional $\mathrm{CT}$ imaging parameters in metastatic renal cell carcinoma patients. Br J Radiol 91:20160795. https:// www.birpublications.org/doi/10.1259/bjr.20160795

19. Hawighorst $H$, Knapstein $P$, Knopp M, et al (1998) Uterine cervical carcinoma: comparison of standard and pharmacokinetic analysis of timeintensity curves for assessment of tumor angiogenesis and patient survival. Cancer Res 58:3598-3602

20. Weidner Net al (1992) Tumor angiogenesis: a new significant and independent prognostic indicator in early-stage breast carcinoma. J Natl Cancer Inst 84:1875-1887. https://doi.org/10.1093/jnci/84.24.1875

21. Weidner N, Carroll PR, Flax J, Blumenfeld W, Folkman J (1993) Tumor angiogenesis correlates with metastasis in invasive prostate carcinoma. Am J Pathol 143:401-409

22. Schoell WM, Pieber D, Reich O et al (1997) Tumor angiogenesis as a prognostic factor in ovarian carcinoma: quantification of endothelial immunoreactivity by image analysis. Cancer 80:2257-2262. https://doi. 
org/10.1002/(SICI) 1097-0142(19971215)80:12<2257::AID-CNCR6>3.0.

$\mathrm{CO} ; 2-\mathrm{R}$

23. Ren J, Liu H, Yan L, Tian S, Li D, Xu Z (2011) Microvessel density and heparanase over-expression in clear cell renal cell cancer: correlations and prognostic significances. World J Surg Oncol 9:158 https://doi.org/110.11 86/1477-7819-1189-1158

24. Yildiz E, Ayan S, Goze F, Gokce G, Gultekin EY (2008) Relation of microvessel density with microvascular invasion, metastasis and prognosis in renal cell carcinoma. BJU Int 101:758-764 https://doi.org/710.1111/j.1464-1410X.2007. 07318.x

25. MacLennan GT, Bostwick DG (1995) Microvessel density in renal cell carcinoma: lack of prognostic significance. Urology 46:27-30. https://doi. org/10.1016/S0090-4295(99)80153-8

26. lakovlev W, Gabril M, Dubinski W, et al (2012) Microvascular density as an independent predictor of clinical outcome in renal cell carcinoma: an automated image analysis study. Lab Investig 92:46-56 https://doi. org/10.1038/labinvest.2011.1153

27. Chen Y, Zhang J, Dai J, Feng X, Lu H, Zhou C (2010) Angiogenesis of renal cell carcinoma: perfusion CT findings. Abdom Imaging 35:622-628 https:// doi.org/610.1007/s00261-00009-09565-00260

28. Wang JH, Min PQ, Wang PJ, et al (2006) Dynamic CT evaluation of tumor vascularity in renal cell carcinoma. AJR Am J Roentgenol 186:1423-1430 https://doi.org/1410.2214/ajr.1404.1408

29. Heng DY, Xie W, Regan MM et al (2009) Prognostic factors for overall survival in patients with metastatic renal cell carcinoma treated with vascular endothelial growth factor-targeted agents: results from a large, multicenter study. J Clin Oncol 27:5794-5799 https://doi.org/5710.1200/ JCO.2008.5721.4809

30. Schwartz LH, Litiere S, de Vries E et al (2016) RECIST 1.1-Update and clarification: from the RECIST committee. Eur J Cancer 62:132-137 https:// doi.org/110.1016/j.ejca.2016.1003.1081

31. Seymour L, Bogaerts J, Perrone A, et al (2017) iRECIST: guidelines for response criteria for use in trials testing immunotherapeutics. Lancet Oncol 18:e143-e152 https://doi.org/110.1016/S1470-2045(1017)30074-30078

32. Donskov F, Jensen NV, Schmidt-Hansen T, Broendum L, Geertsen G (2018) A randomized phase II trial of interleukin-2 and interferon-alpha plus bevacizumab versus interleukin-2 and interferon-alpha in metastatic renal-cell carcinoma (mRCC): results from the Danish Renal Cancer Group (DaRenCa) study-1 (2018). Acta Oncol 57:589-594. https://doi.org/10.1080/0284186X.0282018.1433324

33. Kluger HM, Siddiqui SF, Angeletti C et al (2008) Classification of renal cell carcinoma based on expression of VEGF and VEGF receptors in both tumor cells and endothelial cells. Lab Invest 88:962-972 https:/doi.org/910.1038/labinvest.2008.1065

34. Mertz KD, Demichelis F, Kim R et al (2017) Automated immunofluorescence analysis defines microvessel area as a prognostic parameter in clear cell renal cell cancer. Hum Pathol 38:1454-1462 https://doi.org/1410.1016/j. humpath.2007.1405.1017

35. Puerto-Nevado L, Rojo F, Zazo S et al (2014) Active angiogenesis in metastatic renal cell carcinoma predicts clinical benefit to sunitinib-based therapy. Br J Cancer 110:2700-2707 https://doi.org/2710.1038/bjc.2014.2225

36. Turajilic S, Xu H, Litchfield K et al (2018) Tracking cancer evolution reveals constrained routes to metastases: TRACERx Renal. Cell 173:581-594.e512 https://doi.org/510.1016/j.cell.2018.1003.1057

37. Kammerer-Jacquet SF, Brunot A, Pladys A, et al (2017) Synchronous metastatic clear-cell renal cell carcinoma: a distinct morphologic, immunohistochemical, and molecular phenotype. Clin Genitourin Cancer 15:e1-e7 https://doi.org/10.1016/j.clgc.2016.1006.1007

38. Donskov F, Xie W, Overby A, et al (2020) Synchronous versus metachronous metastatic disease: impact of time to metastasis on patient outcomeresults from the International Metastatic Renal Cell Carcinoma Database Consortium. Eur Urol Oncol 3:530-539 https://doi.org/510.1016/j.euo.2020.1 001.1001

\section{Publisher's Note}

Springer Nature remains neutral with regard to jurisdictional claims in published maps and institutional affiliations.

\section{Submit your manuscript to a SpringerOpen ${ }^{\circ}$ journal and benefit from:}

- Convenient online submission

- Rigorous peer review

- Open access: articles freely available online

- High visibility within the field

- Retaining the copyright to your article

Submit your next manuscript at $\boldsymbol{\nabla}$ springeropen.com 